\title{
A nutrition-focused physical examination workshop improves skills and knowledge of registered dietitians in the diagnosis of malnutrition
}

\author{
N. Dervan ${ }^{1,2}$, B. Gillman ${ }^{3}$, M. McKiernan ${ }^{3}$ and C.A. Corish ${ }^{1,2}$ \\ ${ }^{1}$ School of Public Health, Physiotherapy and Sports Science, University College Dublin, Belfield, Dublin, Ireland, \\ ${ }^{2}$ Institute of Food and Health, University College Dublin, Belfield, Dublin, Ireland and \\ ${ }^{3}$ Mater Misericordiae University Hospital, Eccles Street, Dublin, Ireland
}

A nutrition-focused physical examination (NFPE) is a systematic assessment of nutrition-related health, encompassing a head-to-toe evaluation for micronutrient deficiencies, malnutrition, digestive health and functional status ${ }^{(1,2)}$. Twenty-two CORU-registered dietitians attended an Academy of Nutrition and Dietetics hands-on training workshop on the performance and application of NPFE in the diagnosis of malnutrition in June 2018. The aim of this study was to evaluate the impact of the training on the knowledge and skills of dietitians in performing NFPE and diagnosing malnutrition.

Prior to and at 3, 6, 9 and 12 months following the workshop, the knowledge and skills of each dietitian were evaluated. To assess knowledge and skills, each dietitian answered an anonymous questionnaire and completed a 2-week caseload audit of their practice. Ethics exemption was granted by the Office of Research Ethics in University College Dublin.

Prior to the workshop, only 4 dietitians were performing NFPE, inadequate training and time being cited as the main barriers. All reported performing NFPE after the workshop; however, time remained a barrier to consistent practice. 3 dietitians reported they did not diagnose malnutrition at all prior to the workshop. Of those who diagnosed malnutrition, only 3 and 5 dietitians reported classifying malnutrition as moderate or severe, respectively. After the workshop, all dietitians reported diagnosing the presence and severity of malnutrition. In the caseload audits, NFPE was carried out in $18 \%$ of 509 patients at baseline increasing to $36 \%$ of 368 at 3 months, $33 \%$ of 417 at 6 months, $32 \%$ of 318 at 9 months and $49 \%$ of 212 at 12 months. The percentage of patients diagnosed with malnutrition decreased from $41 \%$ at baseline to an average of $27 \%$ (range $24-30 \%$ ) over the 3, 6, 9 and 12 month audit time points; however, classification of severity of malnutrition increased from $27 \%$ at baseline to an average of $84 \%$ (range $79-90 \%$ ). The use of enteral feeding in malnourished patients consistently increased from $10 \%$ at baseline to an average of $31 \%$ (range 22 $37 \%$ ) at the audit time points. Furthermore, multidisciplinary team interactions for the malnourished cases increased consistently from 2.19 interactions per patient at baseline, to an average of 2.87 interactions (range 2.37-3.67).

The knowledge and skills of dietitians in performing NFPE and diagnosing malnutrition improved following the training workshop. Improved diagnosis of malnutrition severity appeared to increase rates of enteral feeding and collaboration with the multidisciplinary team.

\section{Acknowledgements}

Ms. Beth Mordarski, Ms. Lori Granich, Dr. Anne Griffin, the NFPE trained dietitians and dietitian managers.

\section{References}

1. Mordarski B (2016) J Academy Nutr Diet 116, 868-869

2. White JV, Guenter, Jensen G, et al. (2012) J Academy of Nutr Diet 112 (5), 730-8 